\title{
Improved statistical quark model for the nucleon structure function
}

\author{
Carlos Mírez ${ }^{1}$ \\ Instituto de Física Teórica, Universidade Estadual Paulista, Rua Dr. Bento Teobaldo Ferraz, 271, \\ Barra-Funda, 01140-070, São Paulo, SP, Brazil.
}

\begin{abstract}
An improved statistical quark model, with quark energy levels given by a central linear confining potential, is used to obtain the light sea-quark asymmetry, $\bar{d} / \bar{u}$, and also for the corresponding difference $\bar{d}-\bar{u}$, inside the nucleon. In the model, a temperature parameter is adjusted by recent results obtained for the Gottfried sum rule violation, with two chemical potentials adjusted by the valence up and down quark normalizations. The results are compared with available recent experimental data.
\end{abstract}

Keywords: flavor symmetries, structure function, quark models

PACS: $11.30 . \mathrm{Hv}, 12.39 . \mathrm{x}, 14.65 . \mathrm{Bt}$

We report recent results obtained within a phenomenological statistical quark model for the quark-sea distribution inside the nucleon. This approach provides an improved version of a model presented in Ref. [1], where light $u$ and $d$ quarks have zero mass, with their levels being generated by a relativistic confining model, with a linear scalar plus vector potential. The other parameters in the statistical model, temperature and chemical potentials, are adjusted by recent experimental data.

For the motivation of such a phenomenological approach, we first observe that in the actual theories one cannot find a simple explanation for the observed light sea-quark distribution, $\bar{d} / \bar{u}$, inside the nucleon, compatible with the available experimental data [2]. For instance, by considering only perturbative QCD and gluon splitting processes, the violation of the Gottfried sum rule (GSR) [3] is not reproduced, as the ratio $\bar{d} / \bar{u}$ becomes equal to one, due to the equal probabilities of gluon splittings in $d \bar{d}$ or $u \bar{u}$ pairs. So, by following an idea first presented by Field and Feynman [4], it was shown that the Pauli exclusion principle can be considered in statistical models to obtain different quantities of $\bar{u}$ and $\bar{d}$ in the nucleon sea $[1,5,6,7,8]$.

The statistical quark model [1] is parameterized by the experimental available data, with a temperature parameter adjusted by the GSR violation and two chemical potentials to adjust the valence $u$ and $d$ quarks in the nucleon. All individual quarks of the system, valence and sea quarks, are confined by a central effective interaction, with strength $\lambda_{q}$ ( $q$ stands for the quark flavor) and equal expressions for the scalar and vector components [9], given by $V(r)=(1+\beta) \lambda_{q} r / 2$. With this interaction, a single particle spectrum with energy levels $\varepsilon_{i}$ is generated for the confined quarks by the Schrödinger-like equation, which is derived from the Dirac equation. In the present

\footnotetext{
${ }^{1}$ Report on a work in collaboration with L. Tomio, L.A. Trevisan and T. Frederico
} 
TABLE 1. Evolution of the GSR violation $\left(I_{G S R}\right)$ with the temperature $T$ $(\mathrm{MeV})$ : In the left side we have the results without instanton effects; and, in the right side, by including instanton effects in the fundamental state.

\begin{tabular}{cccc}
\hline$T(\mathbf{M e V})$ & $\mu_{u}$ & $\mu_{d}$ & $I_{G S R}$ \\
\hline 90 & 219 & 157 & 0.016 \\
100 & 171 & 110 & 0.058 \\
110 & 127 & 73 & 0.142 \\
120 & 92 & 49 & 0.242 \\
\hline
\end{tabular}

\begin{tabular}{cccc}
$T(\mathbf{M e V})$ & $\mu_{u}$ & $\mu_{d}$ & $I_{G S R}$ \\
\hline 90 & 193 & 131 & 0.030 \\
100 & 151 & 92 & 0.087 \\
110 & 113 & 63 & 0.174 \\
120 & 83 & 44 & 0.264 \\
\hline
\end{tabular}

approach, we assume $\lambda_{u}=\lambda_{d} \equiv \lambda$, with zero current quark masses for the $u$ and $d$ quarks $\left(m_{u}=m_{d} \equiv m=0\right.$ ), then, the energy levels are the same for both flavors, obtained by using the confining potential model.

With the actual experimental value for the GSR violation [2], we obtain

$$
I_{G S R} \equiv \frac{1}{2}-\frac{3}{2} S_{G}=\int_{0}^{1}(\bar{d}(x)-\bar{u}(x)) d x=0.118 \pm 0.012,
$$

for $T=104 \mathrm{MeV}, \mu_{u}=131 \mathrm{MeV}$ and $\mu_{d}=76 \mathrm{MeV}$. In Table 1 , we show the evolution of the GSR violation with the temperature, given in MeV. As shown, the violation of the GSR is followed by an increasing in the temperature parameter. The results are shown with and without instanton effects in the ground-state energy levels, as prescribed in Ref. [10].

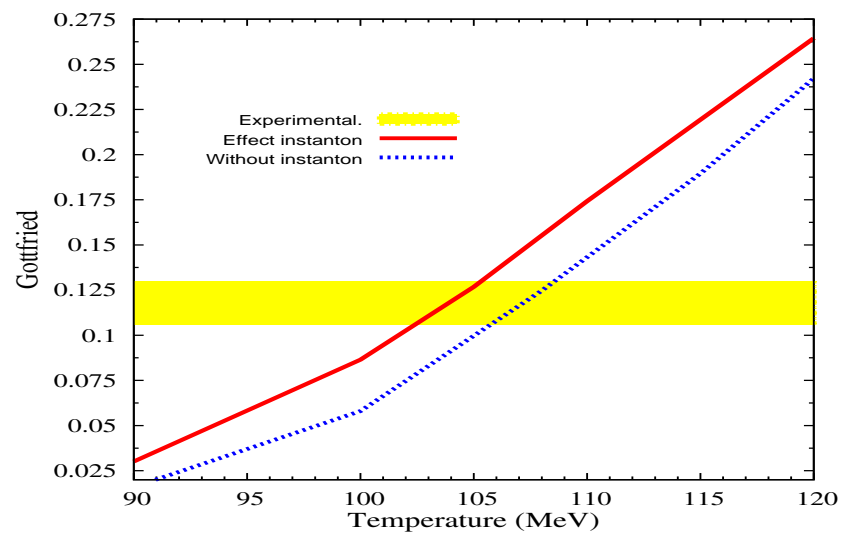

FIGURE 1. Evolution of the value obtained for the GSR violation with the temperature (MeV). The experimental value is obtained from ref.[2]. By considering instanton effects[10], we reproduced the value of $I_{G S R}$ with T=104 MeV and chemical potentials $\mu_{u}=131 \mathrm{MeV}$ and $\mu_{d}=76 \mathrm{MeV}$. Without instanton effects, correspondingly we have $\mathrm{T}=107 \mathrm{MeV}$ with chemical potentials $\mu_{u}=136 \mathrm{MeV}$ and $\mu_{d}=80 \mathrm{MeV}$.

Next, we incorporate in the model contributions obtained from gluonic splitting process, with emission of quark-antiquark pairs. This is a well studied process [11], with equal chances to originate $d \bar{d}$ or $u \bar{u}$ pairs. The probability for such particle-antiparticle pair emissions is given by the Gribov-Lipatov-Altarelli-Parisi equation [12, 13]. We first consider a gluon creation from the original quark distribution, and after the splitting of 
the gluons $[11,14]$. The gluon splitting processes is represented by the parameter $\alpha_{s}$ in the model. The effect resulted by the gluon splitting processes can be observed in the upper frame of Fig.2.

For the constituent quark structure function inside the nucleon we have considered the model given in Ref. [15]. Such function is extracted from the asymptotic form of the pion wave function. Our assumption that it is not changed inside the nucleon. By considering more realistic pion and nucleon wave functions, it is reasonable to think that one can diminish further the relevance of explicit gluon contributions in the model. We verify how the substructure of the constituent quarks can affect the results. Such substructure is obtained from a parametrization presented in Ref. [17] for the structure function of a valence quark in the pion. The parametrization is also detailed in Refs. $[1,16]$, in a convolution model for the constituent quark substructure.
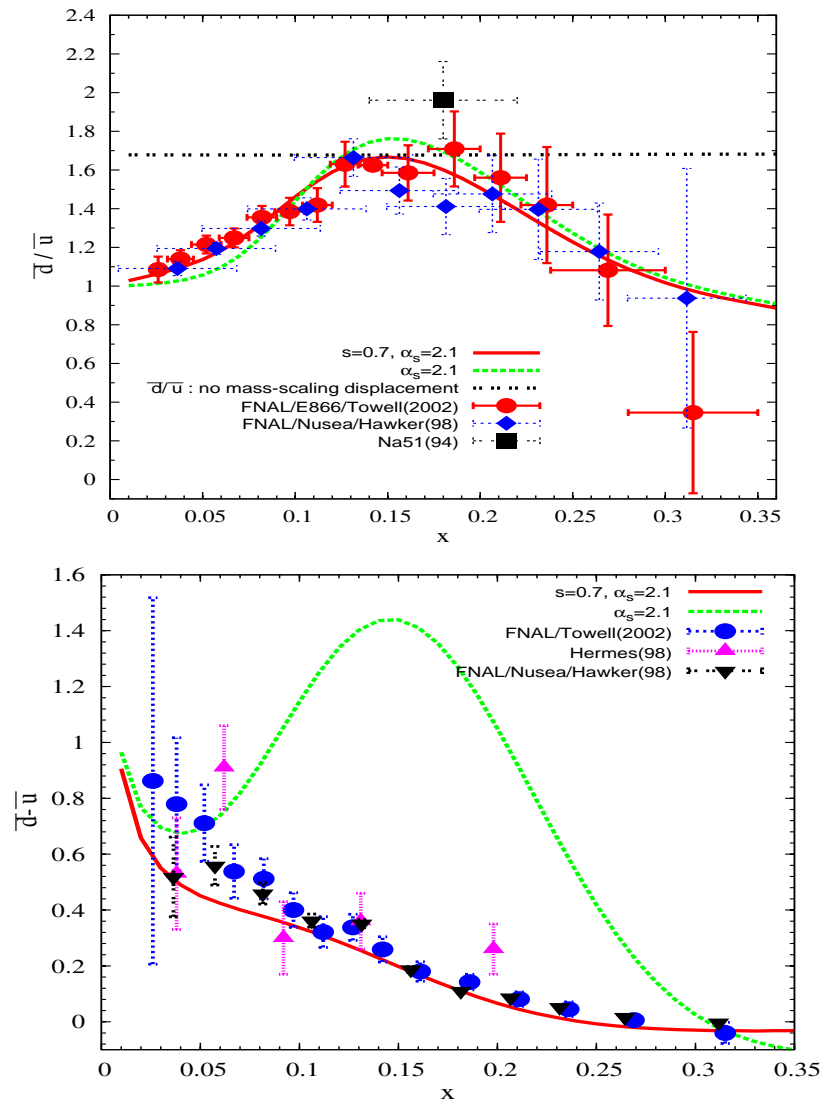

FIGURE 2. The model results for $\bar{d} / \bar{u}$ (upper frame) and $\bar{d}-\bar{u}$ (lower frame), as functions of $x$, compared with data obtained from E866/Towell[2], NuSea/Hawker[18] and Na51[19]. Without mass shift, we obtain the constant dot-dashed-line. With mass-scaling displacement $\left(M_{d} / M_{u}=1.25\right)$, we obtain the other two plots: With dashed-line (green) curve, $\alpha_{s}=2.1$; and, with solid (red) line, $\alpha_{s}=2.1$ and $s=0.7$. 
As shown, such effect is relevant to obtain a good fitting for $\bar{d}-\bar{u}$ and $\bar{d} / \bar{u}$ (see Fig.2). In this way, to obtain a better fitting, we need to combine the values of $\alpha_{s}$ (from the gluon splitting process) with the values of the parameter $s$, corresponding to pionic processes (given by the model of Ref. [17]).

Finally, a simple mathematical trick, based in the Dirac's delta distribution, was used to obtain the shift of the given structure functions, and applied to obtain $\bar{d}(x) / \bar{u}(x)$ ratio inside the proton. This method implies in a an effective mass scaling displacement for the $u$ and $d$ quarks, which is given by $M_{d} / M_{u}=1.25$. For details, see Ref. [1].

In Fig. 2, with solid (red) line, we show the results obtained by considering all the effects: the statistical model, mass shift, gluon splitting, and the contribution from the constituent quark substructure included through the convolution model shown in Ref. [1]. In the upper frame we show the different contributions to the antiquark ratio $\bar{d} / \bar{u}$. In the lower frame, we have the corresponding $\bar{d}-\bar{u}$ in the nucleon sea.

As we have used the same confining potential, in the statistical model we obtain a constant ratio in $\bar{d} / \bar{u}$ (upper frame of Fig.2: point (black) line). The probability effect of gluon emission decreases when the quark substructure is considered, corresponding to $\alpha_{s}$ varying from 2.0 to 2.2 . Indeed, we observed that the probability of gluon emission decreases when we consider the quark substructure.

More details of the results for the ratios and differences of the neutron and proton structure functions, as well as for the corresponding quark-sea distributions are shown in Ref. [16].

I would like to thank my supervisor, Prof. L. Tomio, for discussions on the project and for the assistance in this report. I also want to thank Prof. Tobias Frederico and Dr. Luis Trevisan for discussions. The project, within a doctorate program, is being supported by a fellowship from Conselho Nacional de Desenvolvimento Científico e Tecnológico $(\mathrm{CNPq})$. The collaboration also have partial support from Fundação de Amparo à Pesquisa do Estado de São Paulo.

\section{REFERENCES}

1. L. A. Trevisan, C. Mírez, L. Tomio and T. Frederico, Eur. Phys. J. C 56, 211 (2008).

2. R. S. Towell et al., Phys. Rev. D 64, 052002 (2002).

3. K. Gottfried, Phys. Rev. Lett. 18, 1174 (1967).

4. R. D. Field and R. P. Feynman, Phys. Rev. D15, 2590 (1977).

5. L. A. Trevisan, L. Tomio and T. Frederico, Eur. Phys. J. C 11, 351 (1999).

6. C. Bourrely, F. Buccella, and J. Soffer, Eur. Phys. J. C 23487 (2002); Eur. Phys. J. C41, 327 (2005).

7. J. Cleymans, R. L. Thews, Z. Phys. C 37, 315 (1988).

8. E. Mac e E. Ugaz, Z. Phys. C 43, 655 (1989).

9. P. L. Ferreira, J. A. Helayel, and N. Zagury, N. Cim. A 55, 215 (1980).

10. A. E. Dorokhov, N. I. Kochelev and Yu. A. Zubov, Sov. J. of Part. Nucl. 23, 522 (1992).

11. F. Halzen and A.D. Martin, Quarks and Leptons - An Introductory Course in Modern Particle Physics, Wiley, 1984, pp. 215.

12. G. Altarelli and G. Parisi, Nucl. Phys. B 126, 298 (1977).

13. W. Greiner, Quantum Chromodynamics, Springer, 3ed, 2007.

14. H. R. Christiansen and J. Magnin, Phys. Lett. B 445, 8 (1998). J. Magnin and H. R. Christiansen, Phys. Rev. D 61, 054006 (2000).

15. T. Frederico and G. Miller, Phys. Rev. D 50, 210 (1994).

16. C. Mirez, L.A. Trevisan, T. Frederico, and L. Tomio, preprint, to appear in Nucl. Phys. B, Proceedings.

17. M. Glück, E. Reya, and I. Schiebein, Eur. Phys. J. C 10, 313 (1999).

18. FNAL/E866/NuSea Collab, E.A. Hawker et al., Phys. Rev. Lett. 80, 3715 (1998).

19. Na51 Collab. Phys. Lett. B332, 244 (1994). 
Copyright of AIP Conference Proceedings is the property of American Institute of Physics and its content may not be copied or emailed to multiple sites or posted to a listserv without the copyright holder's express written permission. However, users may print, download, or email articles for individual use. 